\title{
Integration and characterization of solid wall electrodes in microfluidic devices fabricated in a single photolithography step
}

\author{
T. W. Herling, T. Müller, L. Rajah, M. Vendruscolo, and T. P. J. Knowles* \\ Department of Chemistry, University of Cambridge, \\ Lensfield Road, Cambridge CB2 1EW, United Kingdom \\ J. N. Skepper \\ Department of Physiology, Development and Neuroscience, \\ University of Cambridge, Downing Street, Cambridge CB2 3DY, United Kingdom
}

(Dated: November 4, 2018)

\begin{abstract}
We describe the fabrication and characterization of solid 3-dimensional electrodes in direct contact with microfluidic channels, implemented using a single photolithography step, allowing operation in high-dielectric constant media. Incorporation and self-alignment of electrodes is achieved by combining microsolidic approaches with exploitation of the surface tension of low melting point alloys. Thus the metal forms the walls flanking the channel. We show that this approach yields electrodes with a well-defined, reproducible morphology and stable electronic properties when in contact with biochemical buffers. By combining calibration of the electric field with free-flow electrophoresis we quantify the net solvated charges of small molecules.
\end{abstract}

The ability to control electric fields within microfluidic devices is the foundation for many key applications of this technology 111. Approaches exist to use microsolidics techniques 12 in combination with soft lithography to generate electrodes within separate channels that are suitable for use in low-dielectric media such as oils utilized in digital microfluidics 7 ; however, strong electrostatic screening in conductive aqueous media from ionic species present in solution necessitates the use of fundamentally different approaches to generate strong fields in such media. Indeed, direct electric contact between the electrodes and the conductive sample media, such as aqueous buffer solutions, is required to prevent screening at the electrode interface. This requirement presents a challenge for micro-fabrication, and conventional methods for the integration of such elements rely on multistep processes and controlled alignment of such elements to ensure the accurate relative positioning of the fluidic and electronic components of the device 1318. In this Letter, we combine microsolidics techniques with exploitation of the surface tension of molten InBiSn alloy to fabricate 3-dimensional (3D) electrodes in direct contact with aqueous media within microfluidic devices [19, 20. We characterize the electronic properties of the devices and show that this approach allows a stable electrode/solvent interface to be positioned with micrometer precision as shown in Fig. 1(a) to (c). Furthermore, we demonstrate the use of such electrodes in free-flow micro-electrophoresis to determine accurately the charges of molecular species in solution.

In order to create a direct contact between the metal electrodes and electrolyte within microfluidic channels, we generated, using soft-lithography, arrays of pillars to define the position of the electrodes within the
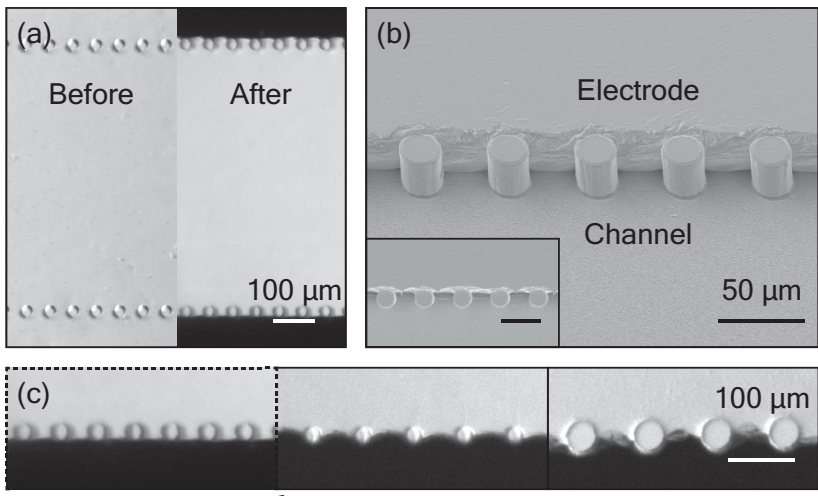

(d)

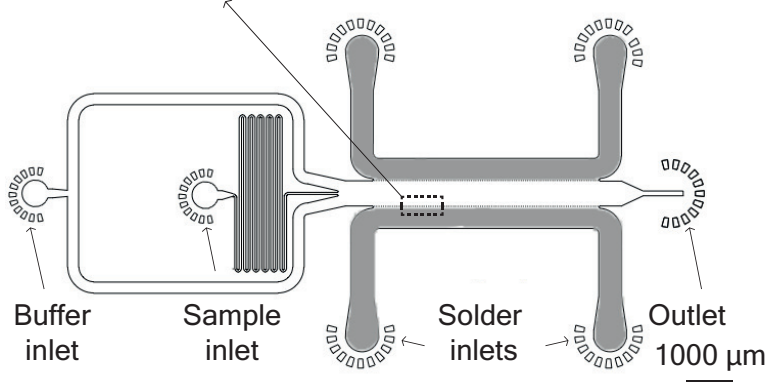

FIG. 1. (a) Brightfield image of the main sample channel before (left) and after (right) electrode incorporation. The channel is flanked by PDMS pillars preventing the solder (opaque) from entering the main channel during device fabrication. (b) Scanning electron micrograph (SEM) of the electrode-channel interface at a $40^{\circ}$ tilt from the position of the glass, below the device. SEM image (insert) of solder and pillars at the channel edge, after the removal of a device from its glass substrate. (c) Variation in pillar spacing and diameter. From left to right; $25 \mu \mathrm{m}$ pillars $25 \mu \mathrm{m}$ apart, $25 \mu \mathrm{m}$ pillars $50 \mu \mathrm{m}$ apart, and $50 \mu \mathrm{m}$ pillars and spacing. (d) The device design used for characterization of the small molecules through. Solder, sample and buffer inlets are indicated, as is the liquid outlet. Electrode channels have been shaded in gray. 
channels[19]. The corresponding sections at the sides of the channel were subsequently filled with a molten InBiSn alloy which was allowed to solidify within the device. Resulting in a large active area and a vertically homogeneous electric field. This is in contrast to conventional approaches where the electrodes are patterned onto the basis of the microchannels.

The microchannels were fabricated in polydimethylsiloxane (PDMS) on glass substrates using standard soft lithography techniques 21. In order to minimize experimental noise, opaque devices were produced by mixing a small fraction of carbon nano powder, $0.2 \% \mathrm{w} / \mathrm{w}$, into the PDMS prior to curing. Electrodes, see Fig. 1(a) and (c), were incorporated by inserting a low melting point InBiSn alloy $(51 \% \mathrm{In}, 32.5 \% \mathrm{Bi}, 16.5 \% \mathrm{Sn}$, Conro Electronics) through the designated channels of devices placed, glass slide down, on a hot plate set to $79^{\circ} \mathrm{C}$. At this temperature, the alloy melts upon contact with the glass substrate and fills the designated channels upon light pressure, but is sufficiently viscous and possesses a sufficient surface tension so as not to flow between the pillars. The inserted electrodes then solidify at room temperature. The fabrication process therefore enables the production of microfluidic devices incorporating 3D electrodes automatically aligned with fluidic components and in direct contact with them.

This approach allows pillar arrays with a range of dimensions to be used in conjunction with the surface tension of molten solder to integrate electrodes in microfluidic devices, as demonstrated in Fig. 1.(c). An upper limit to pillar size and spacing is given by the requirement to prevent the flow of the molten metal between the pillars; in practise effective containment is lost for spacings exceeding $75 \mu \mathrm{m}$. The practical lower limit to pillar sizing is given by the soft lithography process used to define them 21 In the remainder of this Letter, we work with a pillar diameter and spacing of $25 \mu \mathrm{m}$ in each case.

We characterized the stability of the electronic properties of the 3D electrodes in solution and their suitability for applying controlled electric fields in aqueous media within microfluidic devices by repeatedly measuring the frequency-dependent admittance of the device shown in Fig. 1(c) and comparing the results with a suitable circuit model. A scheme of an equivalent circuit for the device is presented in Fig.22(a), where the fluid channel is modeled as a conductance $G$ in series between two solder-to-fluid contacts, characterized by a (low) conductance $G_{C}$ and a parallel capacitance $C_{C}$, with an overall stray capacitance $C_{S}$. To perform the calibration measurement, a $10 \mathrm{mV}$ AC voltage with variable frequency was applied over a device filled with a $500 \mu \mathrm{S} / \mathrm{cm}$ conductivity standard. The resulting current, and thus the admittance $Y$, was then measured as a voltage drop over a $220 \Omega$ resistor using a lock-in amplifier and is shown in Fig. 2(b).

At very low frequencies, the overall conductance is limited by the low conductance of the contacts. At intermediate frequencies of approximately $100 \mathrm{~Hz}$, the conductance through the contact capacitance exceeds the

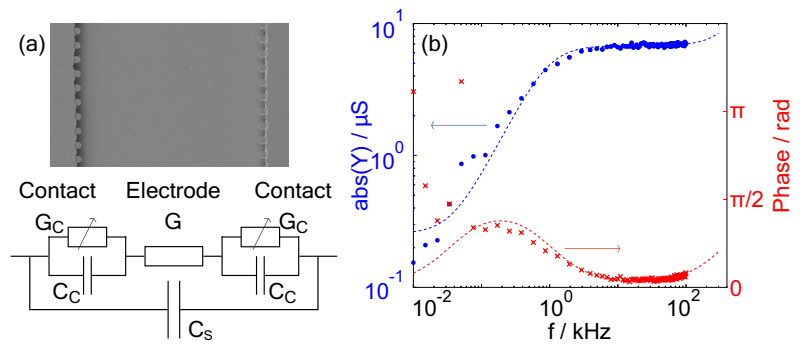

FIG. 2. (a) Top: SEM image of the electrodes and channel taken from the bottom of the channels which are eventually sealed with a glass slide. Bottom: Diagram of the equivalent circuit model of our devices. The conductive channel is represented by a conductor $G$ which is attached to the electrodes via two contacts characterized by a voltage-dependent conductivity $G_{C}$ and a contact capacitance $C_{C}$. The entire device has an additional (stray) capacitance $C_{S}$ in parallel. (b) Measurement of absolute value (blue dots, left axis) and phase (red x-es, right axis) of the admittance $Y$ of a sample device filled with conductivity standard $(500 \mu \mathrm{S} / \mathrm{cm})$ as a function of frequency. The dashed lines are fits to the complex data using the circuit model depicted in (a) with $G, G_{C}$, $C_{C}$ and $C_{S}$ as free parameters.

one through the contact resistance, leading to a linear increase of the admittance with the frequency. Above $1 \mathrm{kHz}$, the conductance of the channel itself becomes the limiting factor governing the overall current flow, and the absolute value of the admittance is constant with frequency until conductance through the stray capacitance becomes appreciable. Comparison to the modeled, complex frequency-dependent admittance (dashed lines in Fig. 2(b) allows for determination of the circuit parameters introduced above, and we obtain contact capacitances of the order of a few $\mathrm{nF}$, stray capacitances in the range of a few $\mathrm{pF}$ and contact resistances much larger than $1 \mathrm{M} \Omega$. Moreover, using the given conductivity of the standard solution thus allows us also to determine the cell constant $K=\sigma / G$ linking conductance $G$ and conductivity $\sigma$ in each device.

Our model fits the experimental data very well, with noticeable deviations only at very low frequencies where conductance is low. Furthermore, since the value of $G$ is directly given by the constant part of the spectrum and is independent of the values of the other fit parameters. The values we obtain for the individual cell constants are highly reliable and reproducible between devices. The mean cell constant and standard deviation for 10 devices were found to be $41 \pm 2.6 \mathrm{~cm}^{-1}$. These results also agree well with calculated values 22, but variation outside the overlap of error bounds were observed when investigating different devices in spite of the shared lithographic dimensions, highlighting the advantage of the direct experimental calibration afforded by this setup.

The functionality of these well-characterized electrodes can be explored by performing quantitative free-flow micro electrophoresis. A key requirement for charge quantification of molecular species by electrophoresis in free 
solution is the ability to determine accurately both the flow profile and the electric field strength across the solution where sample deflection occurs. In order to quantify the electric field inside the flow channel accurately, we use the calibrated cell constants $K$ for the individual devices and measure the conductivities of each of the buffers used. Recording of the DC current flowing through a given device upon application of a voltage then permits the effective voltage drop over the liquid to be determined via $V=I / G$, which in turn yields the electric field through $E=V / w$, with $w=663 \pm 33 \mu \mathrm{m}$ the effective width of the channel[23].

To study the electrophoretic mobility of analytes under controlled conditions of $\mathrm{pH}$ and ionic strength, simultaneous electric current and analyte deflection measurements were undertaken for a range of voltages in three common biochemical buffer systems. Measurements were made in HEPES, Tris and phosphate buffers, each at concentrations of $2.5,5$ and $10 \mathrm{mM}$, and all at $\mathrm{pH}$ 8.0. The fluorescent dyes fluorescein and rhodamine $6 \mathrm{G}$ were chosen as a model analytes.

Experiments were performed at a flow rate of $250 \pm$ $0.88 \mu \mathrm{L} / \mathrm{h}$. The flow velocity in the central $450 \mu \mathrm{m}$ wide section of the channel, averaged in the vertical direction, is to a good approximation constant with lateral position across the channel due to the high aspect ratio. We evaluated the average flow velocity in this areato be $4.97 \pm 0.5 \times 10^{-3} \mathrm{~m} / \mathrm{s}$, resulting in a mean residence time between the electrodes of $0.99 \pm 0.1 \mathrm{~s}$.

The position $\delta$ of the fluorescence intensity maximum of a flow-focused fluorescein or rhodamine beam in different buffer solutions upon application of a DC voltage to the electrodes is shown in Fig. 33(a). Data were recorded using a CCD camera operating through long working distance inverted optics with an integration time of $1 \mathrm{~s}$ and averaged over 25 pixels corresponding to $80 \mu \mathrm{m}$ along the flow direction, with four repeats.

Sample deflection of the order of tens of $\mu \mathrm{m}$ was observed in all buffer systems investigated. However the observed voltage threshold below which ionic screening at the electrode-liquid interface inhibits the current between them, and thereby analyte deflection, varies between the three buffers. Furthermore the voltage drop across the solution relative to that at the metal-liquid interface varies with buffer concentration, affecting the extent of analyte deflection. These observations emphasize that the electric field in the solution is not simply proportional to the overall voltage drop, and highlights the requirement for simultaneous current measurements to calculate the electric field strength from the effective voltage drop over the solution.

When moving close to the electrodes, reaction of the dyes with electrolysis products may result in loss of fluorescence, as seen from the apparent decrease in the deflection data for rhodamine $6 \mathrm{G}$ in 5 and $10 \mathrm{mM}$ Tris at higher applied voltages in Fig. 3. This observation does not affect the dye at locations further from the electrodes, defining the dynamic operating range of the setup.
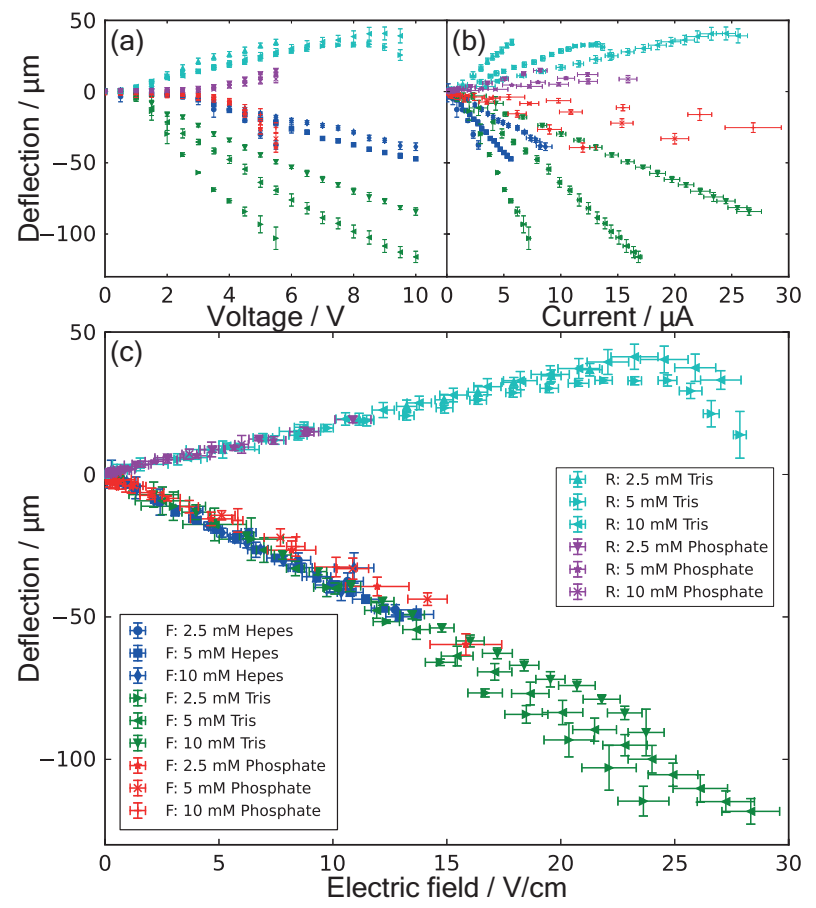

FIG. 3. (a) Analyte deflection $\delta$ measured at the end of the $5 \mathrm{~mm}$ electrode main channel as a function of applied voltage for different buffers. (b) The same deflection data set as in (a) but shown versus the simultaneously measured current through the device. (c) The same deflection measurements as a function of electric field strength $E$ as determined via measured current and calibrated conductance. All vertical error bars represent the statistical spread of averaging the graphical deflection data over 25 pixels in flow direction for 4 repeats. The horizontal error bars are the standard deviation of 4 different repeats (b) and a combination thereof with the uncertainty of the conductivity-to-conductance calibration or calculation (c). The legend in (c) refers to all data shown in this figure. Entries preceded by $\mathrm{F}$ and $\mathrm{R}$ are for data with fluorescein and rhodamine $6 \mathrm{G}$ respectively.

A near-linear dependence of analyte deflection on current is observed when the same deflection data is plotted against the simultaneously measured current in Fig. 3(b). However, due to the differences in buffer conductivities a range of deflections are seen for equal currents. We note that at these low voltages and currents the nucleation and bubble formation of electrolysis products [13, 14, 17] is avoided.

Remarkably, when the deflection is plotted as a function of calibrated electric field in Fig. 3(c), the data collapse accurately into an almost linear relationship, the slope of which defines the electrophoretic mobility $\mu_{p}=v_{p} / E$. To avoid perturbation of $\delta$ by ion depletion due to the low flow velocities at channel edges or reaction with electrolysis products, linear fits were performed to data for field strengths below $15 \mathrm{~V} / \mathrm{cm}$. In this manner, the electrophoretic mobility of the molecular species is found from division of the mean of these slopes by the residence time in the channel $(0.99 \pm 0.1 \mathrm{~s})$ to give elec- 


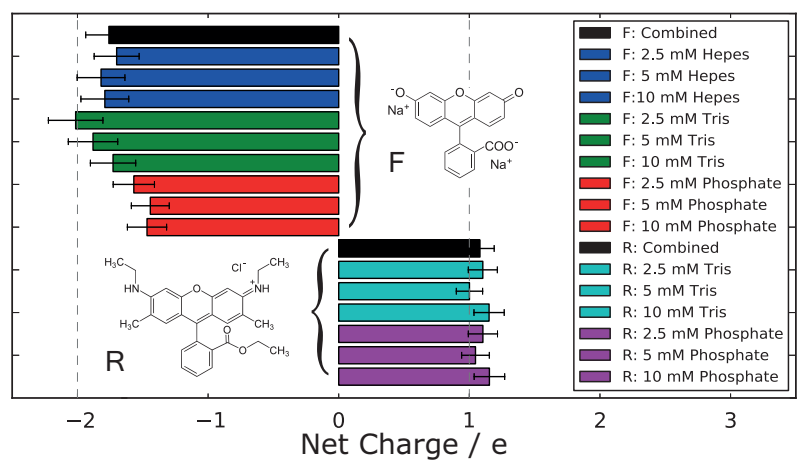

FIG. 4. Net charges determined for fluorescein and rhodamine $6 \mathrm{G}$ using the electrophoretic mobilities found from data for individual buffer systems and the combined mobilities for each dye. The label shows the buffer conditions and dye used, F for fluorescein and $\mathrm{R}$ for rhodamine $6 \mathrm{G}$, and the chemical structures of the two dyes are shown. Dashed vertical lines represent the anticipated charges of -2 (fluorescein) and $+1 e$ (rhodamine 6G), respectively.

trophoretic mobilities of $-3.72 \pm 0.36 \times 10^{-8} \mathrm{~m}^{2} / \mathrm{Vs}$ for fluorescein and $1.75 \pm 0.18 \times 10^{-8} \mathrm{~m}^{2} / \mathrm{Vs}$ in the case of rhodamine $6 \mathrm{G}$. The main contribution to the error on the residence time is the height of the channel $25 \pm 2.5 \mu \mathrm{m}$. All devices used in the experiments presented here were fabricated using the same lithography master, hence variation is not expected between devices.

The measured electrophoretic mobilities can be converted to the corresponding solvated charge for molecules of known radii, here we used $4.5 \AA$ for the radius of fluorescein[25] and $5.9 \AA$ for rhodamine $6 \mathrm{G}[26]$. These values correspond to solvated charges of $-1.8 \pm 0.2 \mathrm{e}$ for fluorescein and $+1.1 \pm 0.1 e$ for rhodamine $6 \mathrm{G}$, see black bars in Fig. 4. These charges determined under solution conditions are in good agreement with those anticipated from the dissociation of the dyes and counter ions shown in the insets of Fig. 4

In conclusion, an adaptable design and fabrication method for the incorporation of solid 3D electrodes of complex geometries into microfluidic devices is presented. A single photolithography step is required in the fabrication of inherently aligned solid electrodes in direct contact with sample solutions. Free-flow electrophoresis was performed in a voltage range where electrolysis products are carried away by the liquid flow through the device. Three buffer systems were characterized at low $\mathrm{mM}$ concentrations. Simultaneous measurements were made of both analyte deflection and current at the application of increasing voltages. The cell constants of the devices were calibrated using conductivity standards. Conductivity measurements of the individual buffer solutions were used to quantify the electric field strength within the electrolyte, enabling the determination of the electrophoretic mobility and the charge of molecules in solution.

Support from the Biotechnology and Biological Sciences Research Council (BBSRC), the Frances and Augustus Newman Foundation, the Engineering and Physical Sciences Research Council (EPSRC) and the Swiss National Science Foundation (SNF) is gratefully acknowledged.
[1] J. Voldman, M. L. Gray, M. Toner, and M. A. Schmidt, Analytical C hemistry 74, 3984 (2002)

[2] T. P. Burg and S. R. Manalis, Applied Physics Letters 83, $2698(2003)$

[3] K. Ahn, C. Kerbage, T. P. Hunt, R. Westervelt, D. R. Link, and D. Weitz, Applied Physics Letters 88, 024104 (2006)

[4] T. P. Burg, M. Godin, S. M. Knudsen, W. Shen, G. Carlson, J. S. Foster, K. Babcock, and S. R. Manalis, Nature 446, 1066 (2007)

[5] D. Kohlheyer, J. Eijkel, A. van den Berg, and R. Schasfoort, Electrophoresis 29, 977 (2008)

[6] M. He and A. Herr, Analytical Chemistry 81, 8177 (2009).

[7] A. Abate, T. Hung, P. Mary, J. Agresti, and D. Weitz, Proceedings of the National Academy of Sciences 107, 19163 (2010)

[8] J. Agresti, E. Antipov, A. Abate, K. Ahn, A. Rowat, J. Baret, M. Marquez, A. Klibanov, A. Griffiths, and D. Weitz, Proceedings of the National Academy of Sciences 107, 4004 (2010).

[9] N. Mojarad and M. Krishnan, Nature Nanotechnology 7, 448 (2012)

[10] B. O'Donovan, D. Eastburn, and A. Abate, Lab on a Chip (2012)
[11] Y. Song, Z. Liu, T. Kong, and H. Shum, Chemical Communications (2013).

[12] A. Siegel, D. Bruzewicz, D. Weibel, and G. Whitesides, Advanced Materials 19, 727 (2007)

[13] D. Kohlheyer, G. A. J. Besselink, S. Schlautmann, and R. B. M. Schasfoort, Lab on a Chip 6, 374 (2006)

[14] S. Kim, J. Kim, K. Kim, and T. Chung, Analytical Chemistry 79, 7761 (2007).

[15] W. Ebina, A. Rowat, and D. Weitz, Biomicrofluidics 3,(2009).

[16] M. B. Romanowsky, M. Heymann, A. R. Abate, A. T. Krummel, S. Fraden, and D. A. Weitz, Lab on a Chip 10, $1521(2010)$

[17] S. Köhler, C. Weilbeer, S. Howitz, H. Becker, V. Beushausen, and D. Belder, Lab on a Chip 11, 309 (2011)

[18] Y. Cheng, X. Luo, C. Tsao, H. Wu, J. Betz, G. Payne, W. Bentley, and G. Rubloff, Lab on a Chip 11, 2316 (2011).

[19] J.-H. So and M. D. Dickey, Lab on a Chip 11, 905 (2011).

[20] S. Li, M. Li, Y. Hui, W. Cao, W. Li, and W. Wen, Microfluidics and Nanofluidics, 1 (2012).

[21] J. McDonald and G. Whitesides, Accounts of Chemical Research 35, 491 (2002).

[22] The calculation was performed by numerically iterating 
the Poisson equation with the potentials of rectangular electrodes $640 \mu \mathrm{m}$ apart as boundary conditions and comparing the applied voltage to the resulting electric field evaluated across the entire length of the device (extending well beyond the end of the electrodes).

[23] The measured width between the faces of the electrodes was $613 \pm 13 \mu \mathrm{m}$. To account for the decreased channel area between the pillars their diameter of $25 \mu \mathrm{m}$ was counted twice and a generous error estimate of $5 \%$ attributed to the conversion from effective voltage drop to electric field.

[24] M. G. Spiga M, International Communications in Heat and Mass Transfer 21, 469 (1994).

[25] D. Montermini, C. Winlove, and C. Michel, The Journal of Physiology 543, 959 (2002).

[26] C. Müller, A. Loman, V. Pacheco, F. Koberling, D. Willbold, W. Richtering, and J. Enderlein, Europhysics Letters 83, 46001 (2008). 